\title{
AZO-POLÍMERO MOLECULARMENTE IMPRESSO COMO ESTRATÉGIA NO DESENVOLVIMENTO DE UMA NOVA PLATAFORMA SENSORIAL PARA DETECÇÃO DE ÁCIDO ÚRICO
}

\section{MOLECULARLY IMPRINTED AZO-POLYMER AS STRATEGY IN THE DEVELOPING A NEW SENSORIAL PLATFORM FOR URIC ACID DETECTION}

Heitor Furlan Trevizan, André Olean-Oliveira, Celso Xavier Cardoso, Marcos F. S. Teixeira

Faculdade de Ciências e Tecnologia - Universidade Estadual Paulista - UNESP.

E-mail: marcos.ff.teixeira@unesp.br

RESUMO - No presente trabalho foi estudado o desenvolvimento de um polímero molecularmente impresso (PMI) baseado no poli(azoBismarck Y) para deteç̧ão de ácido úrico. O filme PMI foi preparado pela eletropolimerização do monômero Bismarck Brown $Y$ na presença de ácido úrico como molécula molde sobre a superfície do eletrodo de óxido de estanho dopado com flúor (FTO). O sensor PMI-poli(azoBismarck Y) exibiu boa resposta eletroquímica, alta sensibilidade e seletividade na ligação específica ao ácido úrico. Sob as condições experimentais otimizadas, a resposta voltamétrica do sensor proposto foi linear no intervalo de concentração de $0,20 \mathrm{mmol} \mathrm{L}^{-1}$ a $0,60 \mathrm{mmol}$ $\mathrm{L}^{-1}$, com limite de detecção de $0,17 \mathrm{mmol} \mathrm{L}^{-1}$.

Palavras chaves: Sensor eletroquímico; polímero molecularmente impresso; detecção de ácido úrico.

ABSTRACT - This present work was studied the development of a molecularly imprinted polymer (MIP) based on the poly(azo-Bismarck $\mathrm{Y})$ for detection of uric acid. The film MIP was electrodeposited on the fluorine-doped tin-oxide (FTO) glass electrode surface by electropolymerization of Bismarck Brown $\mathrm{Y}$ monomer in presence of uric acid as the template molecule. The MIP-poly(azo-Bismarck Y) sensor displays a good electrochemical response, high sensitivity and selectivity in specific binding of the uric acid. Under the optimized experimental conditions, the voltammetric response of the proposed sensor was linear in the concentration range of $0.20 \mathrm{mmol} \mathrm{L}^{-1}$ to 0.60 $\mathrm{mmol} \mathrm{L}^{-1}$, with detection limit of $0.17 \mathrm{mmol} \mathrm{L}^{-1}$.

Keywords: Electrochemical sensor; molecularly imprinted polymer; uric acid detection. 


\section{INTRODUÇÃO}

$O$ ácido úrico $(A U)$ é encontrado no sangue e na urina do ser humano como produto final do metabolismo das proteínas (YUAN et al., 2015). A sua origem no organismo deve-se a quebra das moléculas de purina das proteínas contidas nos alimentos (PEREZ-RUIZ; DALBETH; BARDIN, 2014). A sua variação de concentração na urina e no sangue é tomada como indicativo de possíveis disfunções no organismos e consequentemente um alerta da presença de doenças (BLACK et al., 2018). Dentre algumas doenças relacionadas com níveis anormais de ácido úrico no organismo, destacam-se a hiperuricemia, síndrome de Lesch Nyhan, síndrome metabólica, nefropatia úrica aterosclerose, doença de Parkinson, diabetes do tipo 2 e doença de Alzheimer (PEREZRUIZ; DALBETH; BARDIN, 2014).

Dentro dos métodos analíticos encontrados na literatura para a detecção e quantificação de ácido úrico, existe um número expressivo de trabalhos utilizando biossensores enzimáticos. Porém este tipo de sensores só podem ser usado em condições apropriadas, exigindo controle de $\mathrm{pH}$, temperatura e algumas vezes a adição de cofatores, o que leva a uma baixa estabilidade e baixa reprodutibilidade desses sensores enzimáticos (HWANG et al., 2018). Por outro lado, os sensores eletroquímicos não enzimaticos são considerados uma alternativa para detecção sensível e seletiva para espécies de interesse (ANDRÉ-BARRÈS et al., 2012).

A técnica da impressão molecular surgiu a partir da teoria de Pauling para a formação de anticorpos, onde um antígeno era usado como molécula molde para moldar a cadeia polipeptídica (TARLEY; SOTOMAYOR; KUBOTA, 2005). A partir desta concepção, surgiu a ideia de produzir uma estrutura polimérica rígida tridimensional ao redor de uma molécula molde que pudesse atuar de forma similar ao anticorpo (TALITA et al., 2015; TARLEY; SOTOMAYOR; KUBOTA, 2005). A impressão molecular é uma técnica que tem como objetivo desenvolver polímeros com sítios específicos de reconhecimento da molécula de interesse. Esses polímeros com molécula molde são formados na fase de polimerização, onde ocorre a formação de um complexo entre os monômeros funcionais e a molécula de interesse (ALLENDER et al., 2000; CÉSPEDES; MARTÍNEZ-FÀBREGAS; ALEGRET, 1996). Dessa forma, as terminações dos monômeros funcionais são posicionadas em pontos complementares da molécula alvo, permitindo assim a formação de ligações de hidrogênio e uma notável capacidade enantiosseletiva. Estes complexos são preservados durante a formação da rede polímero reticulados e após sua remoção da rede, deixando seu tamanho, forma, e orientação dos seus sítios de ligação na rede polimérica.

Nas últimas décadas, surgiu um grande esforço no emprego de azo-polímero condutor como plataforma sensorial na construção de sensores químicos. O grande interesse se deve a possibilidade do controle estrutural do polímero, a obtenção de materiais orgânico semicondutores e filmes flexíveis (MERINO; RIBAGORDA, 2012). Alem disso, os filmes poliméricos sintetizado a partir de derivados de azobenzeno apresentam reversibilidade fotoisomérica trans-cis-trans, as quais exibem diferentes propriedades físico-químicas, como também, propriedades de fotorresposta (TEIXEIRA; BARSAN; BRETT, 2016a; YANG et al., 2018). Esse grupo de azo-polímero tem o processo de dopagem que facilita o aumento da condutividade do filme polimérico, isso ocorre pela dispersão ou agregação dos íons dopantes na cadeia polimérica, resultando em bandas eletrônicas livres que influencia no aumento da condutividade (OLEANOLIVEIRA; TEIXEIRA, 2018; TEIXEIRA; BARSAN; BRETT, 2016a).

No presente trabalho estudou-se a formação do polímero molecularmente impresso com o ácido úrico como molécula molde visando o desenvolvimento de um sensor eletroquímico. Os PMIs foram obtidos por eletropolimerização da solução contendo 
o monômero de Bismarck Brown Y e ácido úrico (Figura 1). A otimização do filme polimérico para detecção do ácido úrico foi realizada por voltametria cíclica e voltametria linear.

Figura 1. Estruturas moleculares do monômero Bismarck Brown $Y(A)$ e molde ácido úrico (B).

(A)

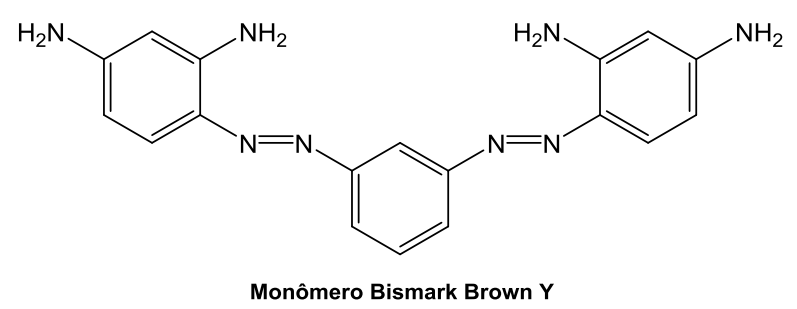

(B)

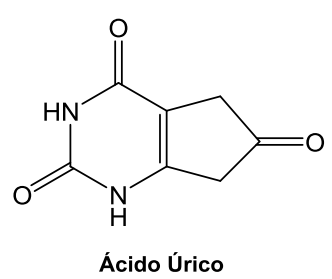

Fonte: autoria própria.

\section{METODOLOGIA}

\subsection{LIMPEZA DO ELETRODO}

Os eletrodos de FTO (óxido de estanho modificado com flúor) foram limpos antes do início do processo de eletropolimerização. Os eletrodos foram submersos em solução de água, hidróxido de amônio e água oxigenada nas proporções de 5:1:1. O eletrodo foi mantido na solução a $70^{\circ} \mathrm{C}$ por $15 \mathrm{~min}$. Posteriormente foram lavados com água destilada, imersos em acetona, sonificados por 3 minutos em banho de ultrassom, lavados novamente com água destilada e secados ao ar.

\subsection{FORMAÇÃO DO POLÍMERO MOLECULARMENTE IMPRESSO (PMI) A PARTIR DO MONÔMERO DE BISMARCK BROWN Y}

O processo de eletropolimerização para formação da PMI a partir do monômero Bismarck Brown $Y$ (BBY) foi conduzido sob superfície de um substrato condutor de FTO de $1,0 \mathrm{~cm}^{2}$ de área geométrica. $A$ eletropolimerização foi realizada em uma célula eletroquímica convencional contendo três eletrodos, sendo: FTO como eletrodo de trabalho, eletrodo de calomelano saturado (ECS) como eletrodo de referência e o eletrodo de fio de platina como eletrodo auxiliar. A célula eletroquímica foi conectada a um potenciostato $\mu$ Autolab type III (Eco Chimie) e gerenciados pelo software NOVA 2.1. O filme em condições otimizadas foi obtido através de voltametria cíclica em solução contendo $10,0 \mathrm{mmol} \mathrm{L}^{-1}$ de BBY e 1,0 $\mathrm{mmol} \mathrm{L}^{-1}$ de ácido úrico em $0,50 \mathrm{~mol} \mathrm{~L}^{-1}$ de $\mathrm{KCl}(\mathrm{pH} 2,0)$, aplicando-se uma velocidade de varredura de $25 \mathrm{mV} \mathrm{s}^{-1}$ e 40 ciclos voltamétricos em um intervalo de potencial de $-0,3$ a $+1,0 \vee$ vs. ECS. As medidas foram realizadas em temperatura ambiente sob atmosfera de $\mathrm{N}_{2}$. Em seguida, para remoção da molécula molde do filme molecularmente impresso sintetizado, o eletrodo modificado foi imerso em $\mathrm{KCl}(\mathrm{pH}=6,9)$ por $15 \mathrm{~min}$ e foram aplicadas varreduras de potenciais $(v=$ $25 \mathrm{mV} \mathrm{s}^{-1}$ ) no intervalo de potencial de $-0,3$ a $+1,0 \mathrm{~V}$ vs ECS até a estabilização dos valores de magnitude de corrente. Para fins comparativos, filmes de polímero não impresso, denominado de PNI, foram sintetizados usando os mesmos parâmetros, mas sem a adição da molécula molde de ácido úrico na solução.

\section{3- COMPORTAMENTO ELETROQUÍMICO E OTIMIZAÇÃO DOS PARÂMETROS ANALÍTICOS DA PMI}

Todas as medidas eletroquímicas foram realizadas em soluções tratadas com borbulho de gás nitrogênio por um tempo mínimo de 15 minutos para exclusão do oxigênio dissolvido. O comportamento eletroquímico do sensor PMI-poli(azo-BBY) foi estudado por voltametria cíclica em solução de $\mathrm{KCl} 0,50 \mathrm{~mol} \mathrm{~L}^{-1}(\mathrm{pH} 6,7)$ na ausência de ácido úrico a $50 \mathrm{mV} \mathrm{s}^{-1}$. Foi estudado a variação de corrente anódica para o eletrodo recoberto com a PMI na ausência e na presença de ácido úrico, através da relação $\Delta I=I_{A U}-I_{0}$, onde $I_{A U}$ é a corrente na presença de ácido úrico e $\mathrm{I}_{0}$ é a corrente na ausência de ácido úrico. 


\section{RESULTADOS E DISCUSSÃO}

\subsection{CONSTRUÇÃO DO SENSOR PMI}

A Figura 2 apresenta o voltamograma cíclico para a eletropolimerização da PMIpoli(azo-Bismarck $Y$ ) sobre a superfície do eletrodo de FTO.

Figura 2. A) Eletropolimerização do PMI sobre eletrodo de FTO em condições otimizadas. Curva vermelha $=$ primeiro ciclo de potencial aplicado. B) Magnificação do voltamograma cíclico do processo de eletropolimerização destacando o primeiro (I) e quadragésimo (II) ciclo de potencial aplicado.
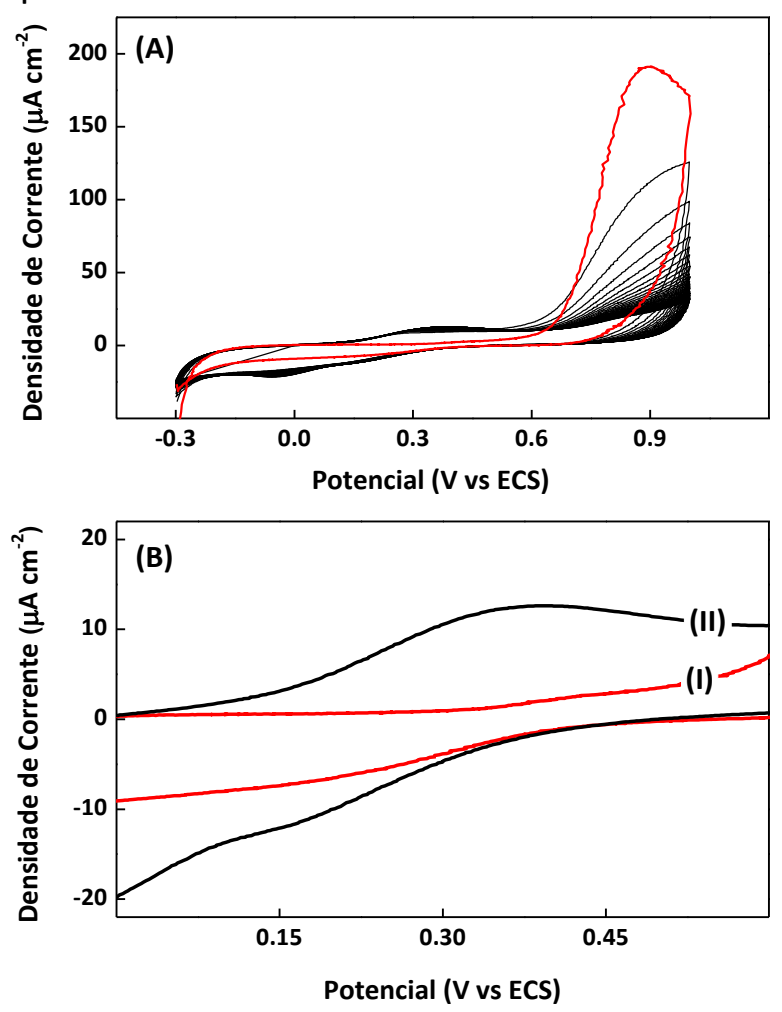

No primeiro ciclo de potencial aplicado, durante a varredura anódica, foi verificado um pico de oxidação em $0,87 \mathrm{~V}$ (vs. ECS) referente a oxidação do monômero de Bismarck Brown $Y$, característico de aminas aromáticas (ELRAHMAN et al., 1991; SEQUOIA; GENIES; TSINTAVIS, 1985; VOLKOV et al., 1980). Nos ciclos subsequentes, houve uma diminuição dos valores de magnitude de corrente no pico de oxidação do monômero e o aumento da magnitude das correntes anódica e catódica, no intervalo de potencial de $-0,30$ a $0,60 \vee$ (vs. ECS), indicando a formação do polímero na superfície do eletrodo de FTO (OLEAN-OLIVEIRA; TEIXEIRA, 2018; REHAN, 2000; TEIXEIRA; BARSAN; BRETT, 2016a). Ao final do 40 으 ciclo de potencial aplicado foi possível evidenciar um par redox com potencial de pico anódico em 0,33 $\mathrm{V}$ e potencial de pico catódico em 0,05 V (vs. ECS), característico do equilíbrio redox de azo-polímeros (REHAN, 2000).

Geralmente a formação dos filmes poliméricos de azobenzeno ocorre pela aplicação de um potencial limite de $+1,0 \mathrm{~V}$ (vs. ECS) para a formação do cátion radical, o que possibilitam o início do processo de eletropolimerização (EL-RAHMAN et al., 1991). O mecanismo de eletroformação do filme polimérico de Bismarck Brown $Y$ está apresentado de forma genérica na Figura 3. O mecanismo é baseado no acoplamento head-to-head, que tem como primeira etapa a oxidação do grupo amina em potencial anódico, formando um cátion radical $\left(\mathrm{BBY}^{\bullet+}\right)$, ou um dicátion $\left(\mathrm{BBY}^{2+}\right)$ em meio ácido (HWANG; SANTHANAM; LIN, 2001).

Figura 3. Mecanismo de polimerização envolvendo formação de radical seguido de acoplamento head-to-head.
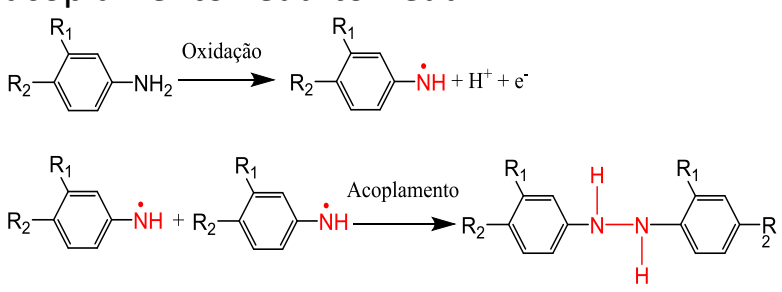

Fonte: Adaptado de (TEIXEIRA; BARSAN; BRETT, 2016b).

A espécie $\mathrm{BBY}^{\bullet+}$ formada reage com um segundo cátion radical em um acoplamento nitrogênio-nitrogênio (headto-head), formando uma ligação covalente, originando o grupo hidrazina. O dímero formado pode sofrer uma nova oxidação dando sequência a formação da cadeia azopolimérica sobre a superfície do eletrodo (HWANG; SANTHANAM; LIN, 2001; LI et al., 2002). O ácido úrico presente na solução de eletropolimerização possui grupos 
nitrogenados e oxigenados capazes de interagir com o monômero de BBY através de ligação de hidrogênio, formando um complexo com o ácido úrico (Figura 4) (AGHAEI; MILANI HOSSEINI; NAJAFI, 2010). A mistura formada é comumente denominado de complexo pré-polimerização (AGHAEl; MILANI HOSSEINI; NAJAFI, 2010; KARIMIAN; GHOLIVAND; TAHERKHANI, 2015). Tais complexos, formados através das ligações de hidrogênio e interações eletrostáticas, são responsáveis por permitir o crescimento da cadeia azo-polimérica em torno das moléculas de ácido úrico, usando-as como molde para a construção dos sítios de ligação específicos para molécula de interesse.

Figura 4. Representação esquemática das possíveis interações e ligações de hidrogênio formadas entre o poli(azo-BBY) e a molécula de ácido úrico.

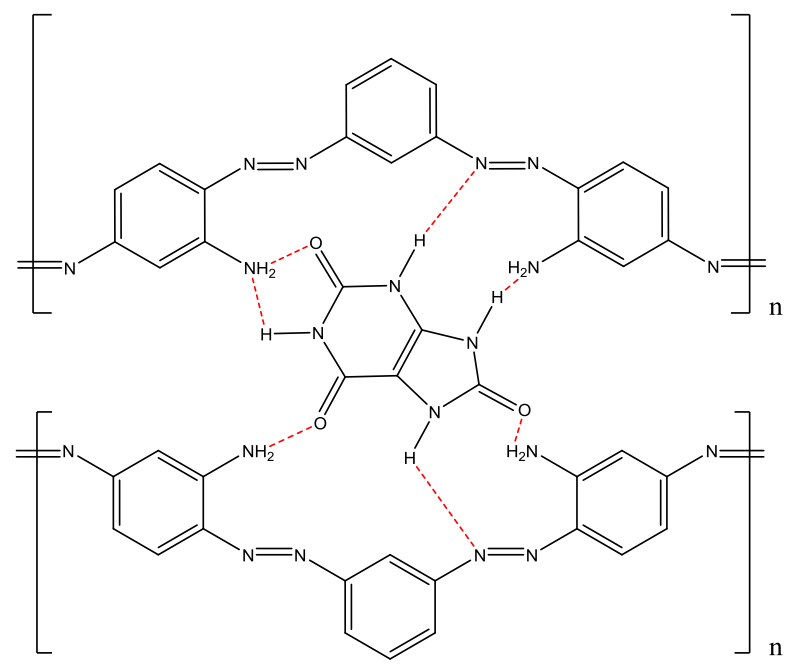

Fonte: Adaptado de (AGHAEI; MILANI HOSSEINI; NAJAFI, 2010).

Após a eletropolimerização do filme de PMI-poli(azo-BBY), o ácido úrico usado como molde é retirado da matriz polimérica por meio da aplicação de ciclagens de potenciais em solução de $\mathrm{KCl} 0,5 \mathrm{~mol} \mathrm{~L}^{-1}(\mathrm{pH}$ $6,7)$ até a completa estabilização dos valores de corrente. A representação esquemática das etapas de construção do sensor baseado em PMI está apresentada na Figura 5.
Figura 5. Representação esquemática do processo de construção do sensor PMIpoli(azo-Bismarck Y).
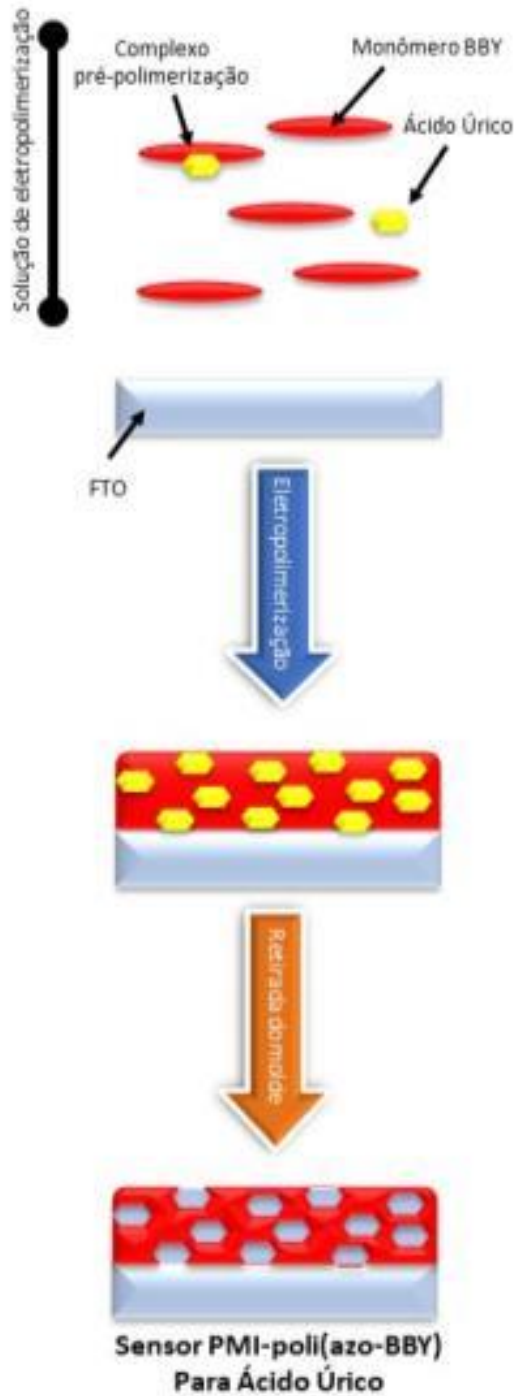

Fonte: autoria própria.

\subsection{COMPORTAMENTO ELETROQUÍMICO DO SENSOR}

De modo a confirmar a formação dos sítios de ligação específico do PMI-poli(azoBismarck Y) com a molécula de ácido úrico, foram realizadas medidas de voltametria cíclica com os eletrodos de PMI-poli(azo$B B Y)$ e PNI-poli(azo-BBY) na ausência e na presença de $1,0 \mathrm{mmol} \mathrm{L}^{-1}$ de ácido úrico (Figura 6). A resposta voltamétrica para o eletrodo de PMI-poli(azo-BBY) na ausência de ácido úrico não apresentou comportamento redox relacionado a oxidação do ácido úrico (Figura 6A). Já na presença da molécula de ácido úrico foi verificado um pico de potencial de oxidação 
em $0,60 \quad V$ vs ECS (Figura 6B) que corresponde a oxidação eletroquímica do ácido úrico.

Figura 6. Voltamogramas cíclico do (A) polímero molecularmente impresso (PMI) e (B) não impresso (PNI) em $\mathrm{KCl} 0,5 \mathrm{~mol} \mathrm{~L}^{-1}$ na ausência e na presença de $1,0 \mathrm{mmol} \mathrm{L}^{-1}$ de ácido úrico (curva vermelha). $v=50 \mathrm{mV} \mathrm{s}^{-1}$.
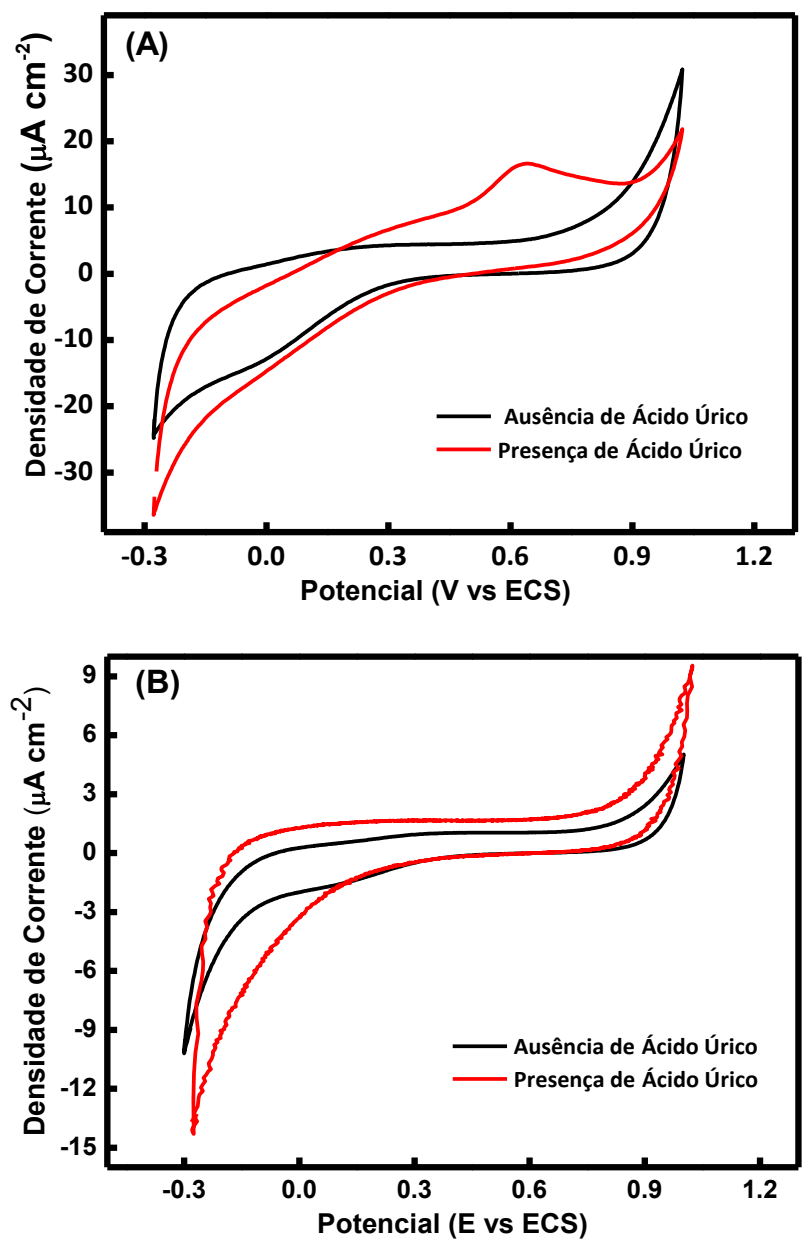

Por outro lado, o filme de PNIpoli(azo-BBY) não apresentou nenhum processo redox em ambas as condições experimentais e não foi verificada a presença do pico de potencial característico da oxidação de ácido úrico. Dessa maneira, foi constatada uma relação direta da oxidação do ácido úrico com a presença dos sítios de ligação específicos presentes no filme MIP-poli(Azo-BBY). A molécula de ácido úrico, ao acessar o sítio vazio de ligação específico torna a gerar o complexo [PMI-poli(azo-Bismarck Y) - ácido úrico] através de ligações de hidrogênio que resultam na diminuição da energia necessária para a oxidação da molécula de ácido úrico presente no sítio ativo (Figura 4) (BARROS; CUSTODIO; RATH, 2016; KARIMIAN; GHOLIVAND; TAHERKHANI, 2015; TORKASHVAND; GHOLIVAND; TAHERKHANI, 2015). O mecanismo completo, bem como as ligações/interações entre o azo-polímero e a molécula de ácido úrico em solução ainda não foram totalmente elucidadas. Entretanto, estudos teóricos e experimentais serão conduzidos afim de se entender de maneira satisfatória a natureza físico-química dessa interação. Por outro lado, nenhuma resposta eletroquímica para ácido úrico foi observada para o eletrodo recoberto com o polímero não impresso. Indicando a ausência dos sítios de ligação específicos no polímero e consequentemente a baixa a interação e estabilização com a molécula de ácido úrico.

\section{3- OTIMIZAÇÃO DO SENSOR BASEADO EM POLÍMERO MOLECULARMENTE IMPRESSO DE POLI(AZO-BBY)}

Com a finalidade de alcançar o melhor desempenho analítico na detecção e quantificação seletiva de ácido úrico, estudos para otimização dos parâmetros de construção do sensor pela técnica de eletropolimerização foram estudados. Nesses estudos, buscou-se refinar os parâmetros com o objetivo de obter maior sensibilidade do dispositivo por meio de maiores valores de variação de corrente de pico anódico $(\Delta /$ anódico $)$. Para isto, parâmetros como velocidade de varredura, número de ciclos e pH na etapa de eletropolimerização foram estudados.

Sabe-se que a taxa de velocidade de varredura influencia significativamente a morfologia dos filmes eletrodepositados. Uma vez que a velocidade de varredura influencia no gradiente de concentração de espécies na interface eletrodo/solução, como também, na taxa eletrônica dos processos eletroquímicos podendo alterar a cinética de polimerização (HOSU et al., 2017)(HOSU et al., 2017). Na Figura 7, são apresentados os valores de variação de 
corrente anódica na presença e na ausência de $1,0 \mathrm{mmol} \mathrm{L}^{-1}$ de ácido úrico para os filmes construídos com diferentes velocidades de varredura aplicada. Por meio da resposta voltamétrica, verificou-se que o PMIpoli(azo-Bismarck Y) sintetizado com taxa de varredura de $25 \mathrm{mV} \mathrm{s}^{-1}$ apresentou melhor magnitudes de corrente de oxidação do ácido úrico em comparação aos filmes construídos com velocidades de varredura superiores.

Figura 7. Gráfico da variação do pico de corrente anódica dos sensores PMI construídos com diferentes velocidades de varredura. As medidas voltametricas foram realizadas em solução de $\mathrm{KCl} 0,50 \mathrm{~mol} \mathrm{~L}^{-1}$ na ausência e na presença de $1,0 \mathrm{mmol} \mathrm{L}^{-1}$ de ácido úrico a $50 \mathrm{mV} \mathrm{s}^{-1}$. A barra de erro representa o desvio padrão para três experimentos feitos separadamente com o mesmo sensor.

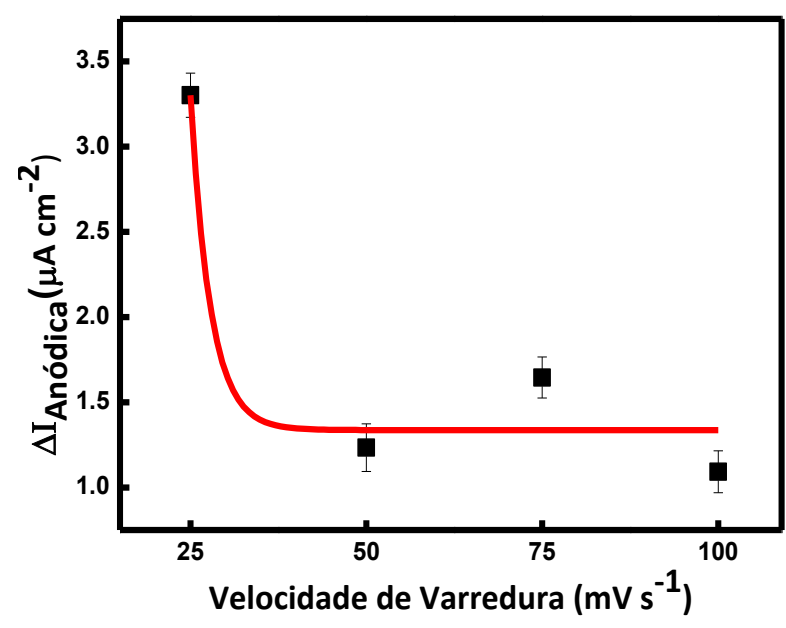

O comportamento da diminuição da magnitude de corrente em uma função exponencial da velocidade de varredura na eletropolimerização revela uma limitação da formação do filme por mecanismo de difusão (HJELM et al., 2004; LEVI et al., 1997).

A resposta eletroquímica dos filmes de PMls construídos são dependentes do número de sítios de ligação específicos para o ácido úrico gerados no polímero durante a etapa de eletropolimerização. Portanto, maiores velocidades de varredura proporcionam uma menor quantidade de sítios de ligação devido a limitação da difusão dos monômeros de BBY e do ácido úrico para a superfície do eletrodo.

o número de ciclos de eletropolimerização também é um fator importante obtenção de filmes poliméricos, determinando tanto a espessura quanto o número de sítios de ligação formados (DEHGHANI; NASIRIZADEH; YAZDANSHENAS, 2019). Para número de ciclos menores do que o ideal, um filme de polímero molecularmente impresso com menos locais de impressão de ligação específica seria criado na superfície do eletrodo. Entretanto, filmes poliméricos com grande densidade poderão obstruir as cavidades moleculares, impedindo $o$ acesso do analito $e$ consequentemente diminuindo $O$ sinal analítico (DEHGHANI; NASIRIZADEH; YAZDANSHENAS, 2019; MOTIA et al., 2018).

A Figura 8 apresenta a resposta anódica para ácido úrico em função do número de ciclos de eletropolimerização. Foi observado que a partir do 40 ciclo de potencial aplicado houve uma diminuição na magnitude da corrente de oxidação do ácido úrico.

Figura 8. Gráfico da variação do pico de corrente anódica para os diferentes sensores PMI construídos com diferentes números de ciclos de potenciais na etapa de eletropolimerzação. Solução de $0,50 \mathrm{~mol} \mathrm{~L}^{-1}$ de $\mathrm{KCl}$ na presença e ausência de $1,0 \mathrm{mmol}$ $\mathrm{L}^{-1}$ de ácido úrico. $v=50 \mathrm{mV} \mathrm{s}^{-1} . \mathrm{N}=3$.

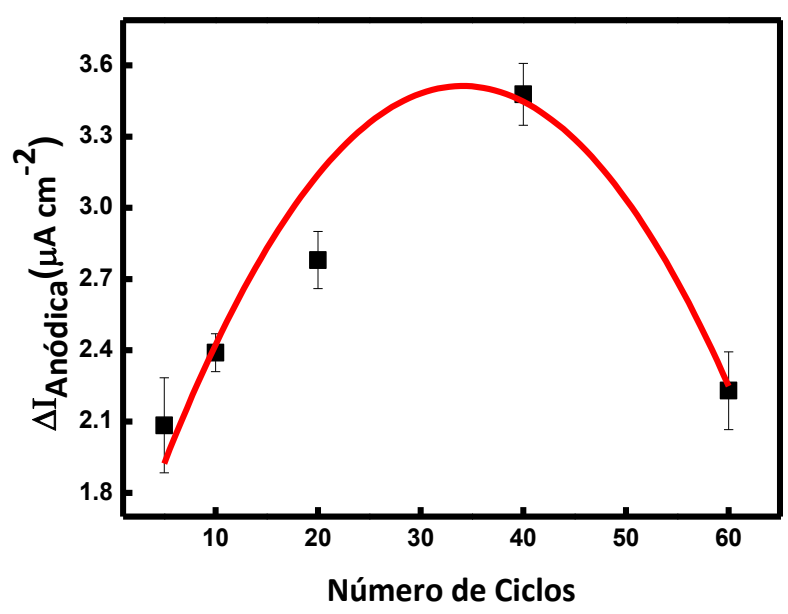


Aplicando-se um número de ciclos inferior a 40, há a formação de um número insuficiente de sítios de ligação. Por outro lado, aplicando-se números de ciclos superiores a 40 ciclos há a obstrução dos sítios de ligação presentes no interior do filme polimérico, formando uma região inativada que diminui $o$ desempenho eletroquímico do sensor PMI (Figura 9).

$\mathrm{O}$ valor do $\mathrm{pH}$ é outro fator importante que afeta a capacidade de reconhecimento molecular e a resposta da corrente de pico. Os valores de $\mathrm{pH}$ utilizado na etapa de eletropolimerização variaram de 2,0 a 8,0. A variação de pico anódico referente a resposta eletroquímica dos filmes obtidas nos diferentes pHs são apresentadas na Figura 10.

Figura 9. Representação esquemática da influência do número de ciclos de potenciais aplicados na construção do filme MIP na reposta eletroquímica frente ao analito de interesse.
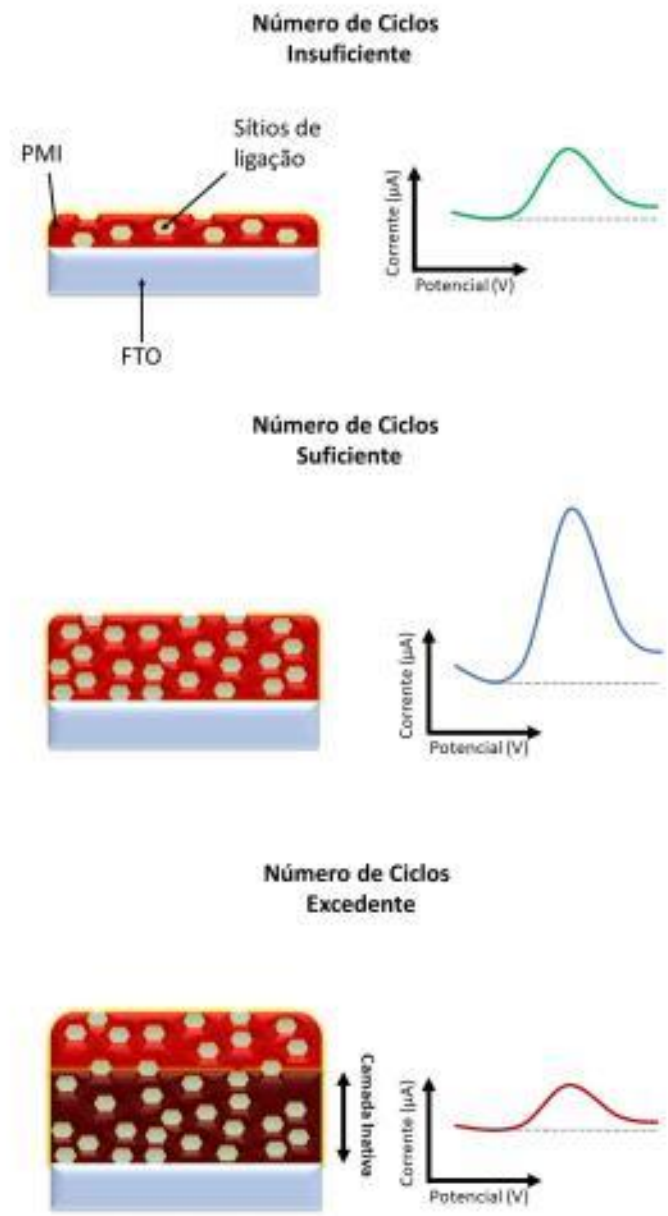

Fonte: autoria própria.
O resultado obtido para os filmes de MIP-poli(azo-BBY) frente a molécula de ácido úrico demonstra que a melhor resposta eletroquímica obtida foi o filme polimérico formado em $\mathrm{pH}$ 2. Para filmes poliméricos formados em valores de $\mathrm{pH}$ superior a 2, o sinal analítico para o ácido úrico diminui expressivamente.

Figura 10. Gráfico da variação do pico de corrente anódica para os diferentes sensores $\mathrm{PMI}$ construídos com diferentes $\mathrm{pHs}$ na eletropolimerzação. Medidas voltametricas realizadas em solução de $\mathrm{KCl} 0,50 \mathrm{~mol} \mathrm{~L}^{-1}$ na presença e ausência de $1,0 \mathrm{mmol} \mathrm{L}^{-1}$ de ácido úrico a $50 \mathrm{mV} \mathrm{s}^{-1} . \mathrm{N}=3$.

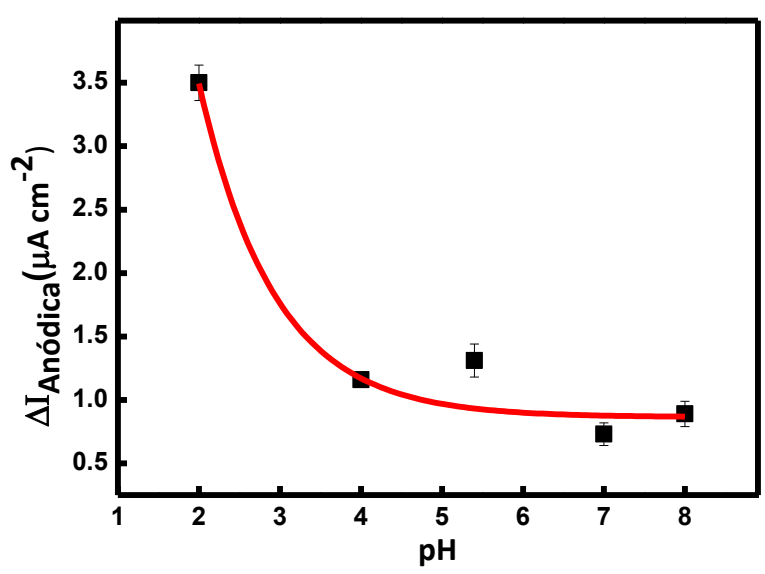

O ácido úrico apresenta formas tautoméricas ceto-enólicas (Figura 11) que são influenciados pelo $\mathrm{pH}$ do meio reacional. Para $\mathrm{pH}$ menores do que 2,0, o ácido úrico se apresenta principalmente em sua forma enol (ZHENG et al., 2018).

Figura 11. Estrutura molecular do equilíbrio ceto-enólico da molécula de ácido úrico.

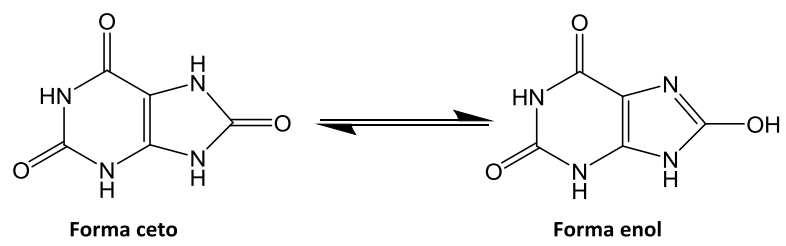
Fonte: autoria própria.

O grupo enol hidrogenado na forma do ácido úrico viabiliza a ligação de hidrogênio e possibilita a interação com o nitrogênio nos sítios de ligação do PMIpoli(azo-Bismarck Y) (ZHENG et al., 2018). Essa interação da ligação de hidrogênio 
ajuda na estabilização do ácido úrico dentro da matriz polimérica e acelera a transferência de elétron do ácido úrico alvo para a superfície do eletrodo e consequentemente aumentando as correntes de pico anódico. À medida que os valores de $\mathrm{pH}$ aumentaram em direção a região básica, o ácido úrico apresenta como um ânion monobásico (ZHENG et al., 2018). Como também nessa faixa de $\mathrm{pH}$, a camada de poli(Azo Bismarck Y), torna-se cada vez mais neutra devido à desprotonação de BBY (pKa $=5,6)$. Nessas condições, são afetados significativamente as interações entre o sítio de ligação do polímero e a molécula de ácido úrico, resultando em uma diminuição da resposta eletroquímica do sensor.

\subsection{DESEMPENHO ANALÍTICO DO SENSOR}

Após otimização na preparação da plataforma sensorial, o desempenho analítico do sensor proposto foi avaliado por curva analítica para o ácido úrico. A resposta do sensor PMI foi obtida por meio de medidas de voltametria de varredura linear em solução de $\mathrm{KCl} 0,5 \mathrm{~mol} \mathrm{~L}^{-1} \mathrm{com} \mathrm{pH} 6,7.0$ desempenho analítico de sensor de PMI foi avaliada utilizando sucessivas adições de padrão de ácido úrico compreendendo o intervalo de concentrações de 0,20 a $1,0 \mathrm{mmol} \mathrm{L}^{-1}$. $\mathrm{O}$ ácido úrico pode ser detectado usando o método do sensor PMI diretamente pela intensidade de corrente do pico anódico em potencial de $0,68 \mathrm{~V}$ (vs. ECS). A Figura 12 apresenta a resposta de voltametria linear e a curva analítica obtida para o sensor PMI-poli(azo-Bismarck Y) após sucessivas adições de padrão de ácido úrico. Uma relação linear $(r=0,9994)$ entre a variação de corrente anódica ( $\Delta \mathrm{l}_{\text {anódica }}$ ) e concentração de $A U$ foi obtida na faixa linear de 0,20 a $0,60 \mathrm{mmol} \mathrm{L}^{-1}$ com uma equação de regressão linear (eq 1.):

$$
\Delta \mathrm{I}(\mu \mathrm{A})=4,24 \pm 0,12[\mathrm{AU}]\left(\mathrm{mmol} \mathrm{L}^{-1}\right)(\mathrm{n}=6)
$$

O limite de detecção (LOD) do método foi calculado com base dos parâmetros da curva analítica utilizando a equação $L O D=$ 3s/b, onde 's' é o desvio padrão estimado de fundo e 'b' é a inclinação da curva (MUCIO et al., 2017). O limite de detecção calculado foi de $0,17 \mathrm{mmol} \mathrm{L}^{-1}$ para o ácido úrico.

Figura 12. A) Resposta em voltametria linear do sensor MIP-poli(azo Bismarck Y) frente a diferentes concentrações de ácido úrico em solução de $\mathrm{KCl} 0,50 \mathrm{~mol} \mathrm{~L}^{-1}$. B) Curva analítica obtida a partir das correntes de pico anódica correspondentes aos voltamogramas. $v=50 \mathrm{mV} \mathrm{s}^{-1} . \mathrm{N}=3$
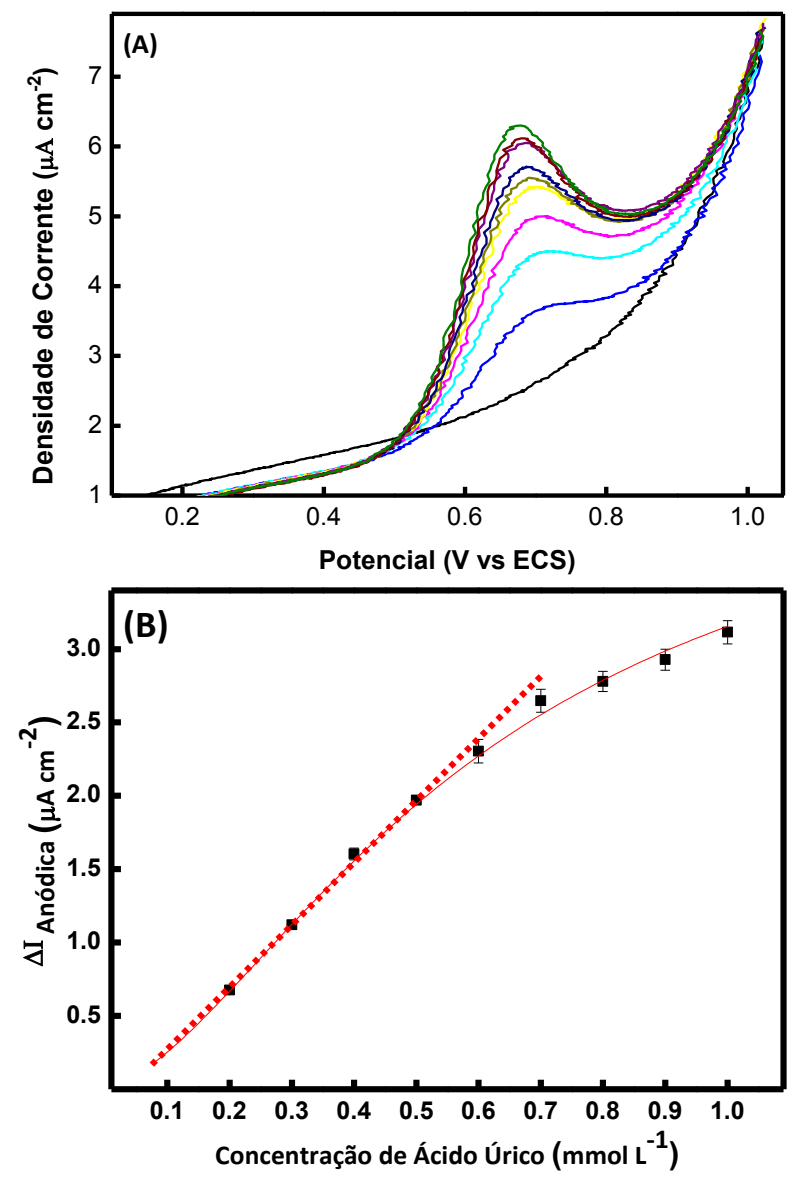

A repetibilidade do procedimento de fabricação foi avaliada fazendo o sensor para cinco repetições na concentração de $A U$ de $1,0 \mathrm{mmol} \mathrm{L}^{-1}$. A corrente de pico média voltametria de varredura linear para estes ensaios foi de $3,53 \mu \mathrm{A} \pm 0,022$. Para testar a reprodutibilidade, cinco sensores foram preparados em cinco dias consecutivos. Os testes repetibilidade foram realizados por voltametria linear em solução de $\mathrm{KCl} 0,5 \mathrm{~mol}$ $\mathrm{L}^{-1} \mathrm{KCl}$ contendo $1,0 \mathrm{mmol} \mathrm{L}^{-1}$ de $\mathrm{AU}$. Os resultados obtidos de cinco medidas replicadas mostraram um desvio de $\pm 0,30$ 
para os valores de correntes anódica. Para examinar a estabilidade de cada eletrodo, os voltamogramas dos sensores PMI em solução de $\mathrm{KCl}(\mathrm{pH} 6,7)$ contendo $1,0 \mathrm{mmol}$ $\mathrm{L}^{-1}$ de $\mathrm{AU}$ foram repetidos durante 15 dias e depois comparados com os dados primários. Os picos atuais do $A U$ no sensor otimizado poderiam reter $85,0 \%$ de seu valor original, implicando que o sensor otimizado exibe uma estabilidade satisfatória na detecção do $\mathrm{AU}$.

\section{CONCLUSÃO}

Neste trabalho, um novo sensor PMI foi desenvolvido para determinação eletroquímica de ácido úrico. Este sensor PMI exibe alta sensibilidade e reconhecimento específico à molécula de ácido úrico, devido à presença de cavidades impressas complementares à molécula molde no filme PMI. O intervalo de resposta linear do sensor para as concentrações de ácido úrico foi $0,20 \mathrm{mmol} \mathrm{L}^{-1}$ a $0,60 \mathrm{mmol} \mathrm{L}^{-1}$ com limite de detecção de $0,17 \mathrm{mmol} \mathrm{L}^{-1}$. 0 bom desempenho eletroquímico do sensor para o analito de interesse indica uma possível aplicação na análise do ácido úrico em amostras de urina. Os materiais para construção da plataforma sensorial apresentam baixo custo relativo e a molécula molde pode ser facilmente removida por voltametria cíclica, não sendo necessário o emprego de nenhum reagente de extração.

\section{AGRADECIMENTOS}

Os autores agradecem a CNPQ/PIBIC (156365/2018-9) e a CAPES pelas bolsas concedidas para H.F.T. e A.O.O., respectivamente. SJT $\Delta$

\section{REFERÊNCIAS}

AGHAEI, A.; MILANI HOSSEINI, M. R.; NAJAFI, $M$. A novel capacitive biosensor for cholesterol assay that uses an electropolymerized molecularly imprinted polymer. Electrochimica Acta, v. 55, n. 5, p. 1503-1508, 1 fev. 2010. https://doi.org/10.1016/i.electacta.2009.09.0 $\underline{33}$
ALLENDER, C. J. et al. Pharmaceutical applications for molecularly imprinted polymers. International Journal of Pharmaceutics, v. 195, n. $1-2$, p. 39-43, $2000 . \quad \quad$ https://doi.org/10.1016/S03785173(99)00355-5

ANDRÉ-BARRÈS, C. et al. Comparison of diffusivities data of streptocyanine dyes by electrochemical and NMR-DOSY methods. v. 686, p. 54-57, 2012. https://doi.org/10.1016/j.jelechem.2012.09. $\underline{030}$

BARROS, L. A.; CUSTODIO, R.; RATH, S. Design of a new molecularly imprinted polymer selective for hydrochlorothiazide based on theoretical predictions using Gibbs free energy. Journal of the Brazilian Chemical Society, v. 27, n. 12, p. 2300-2311, 2016. https://doi.org/10.5935/0103-

$\underline{5053.20160126}$

BLACK, C. N. et al. Uric acid in major depressive and anxiety disorders. Journal of Affective Disorders, v. 225, n. March 2017, p. 684-690, 2018. https://doi.org/10.1016/j.jad.2017.09.003

CÉSPEDES, F.; MARTÍNEZ-FÀBREGAS, E.; ALEGRET, S. New materials for electrochemical sensing I. Rigid conducting composites. TrAC - Trends in Analytical Chemistry, v. 15, n. 7, p. 296-304, 1996. https://doi.org/10.1016/01659936(96)00042-8

DEHGHANI, M.; NASIRIZADEH, N.; YAZDANSHENAS, M. E. Determination of cefixime using a novel electrochemical sensor produced with gold nanowires/graphene oxide/electropolymerized molecular imprinted polymer. Materials Science and Engineering C, v. 96, p. 654-660, 2019. https://doi.org/10.1016/j.msec.2018.12.002

EL-RAHMAN, H. A. A. et al. Oxidative polymerization of $p$-aminoazobenzene in acetonitrile. A new electroactive polymer. 
Journal of Electroanalytical Chemistry, v. 315, n. 1-2, p. 161-174, 1991. https://doi.org/10.1016/0022-

\section{$\underline{\text { 0728(91)80067-Z }}$}

HJELM, J. et al. Electropolymerisation dynamics of a highly conducting metallopolymer: $\quad$ Poly-[Os(4'-(5-(2,2'bithienyl))-2,2':6', 2"-terpyridine)2]2+. Electrochemistry Communications, v. 6, n. 2, p. 193-200, 2004. https://doi.org/10.1016/i.elecom.2003.11.01 $\underline{1}$

HOSU, O. et al. Nanostructured electropolymerized poly(methylene blue) films from deep eutectic solvents. Optimization and characterization. Electrochimica Acta, v. 232, p. 285-295, 2017.

https://doi.org/10.1016/j.electacta.2017.02.1 $\underline{42}$

HWANG, B. J.; SANTHANAM, R.; LIN, Y. L. Nucleation and growth mechanism of electroformation of polypyrrole on a heattreated gold/highly oriented pyrolytic graphite. Electrochimica Acta, v. 46, n. 18, p. 2843-2853, 2001. https://doi.org/10.1016/S00134686(01)00495-9

HWANG, D. et al. Analytica Chimica Acta Recent advances in electrochemical nonenzymatic glucose sensors e A review. Analytica Chimica Acta, v. 1033, p. 1-34, 2018.

https://doi.org/10.1016/i.aca.2018.05.051

KARIMIAN, N.; GHOLIVAND, M. B.; TAHERKHANI, F. Computational design and development of a novel voltammetric sensor for minoxidil detection based on electropolymerized molecularly imprinted polymer. Journal of Electroanalytical Chemistry, v. 740, p. 45-52, 2015. https://doi.org/10.1016/i.jelechem.2014.12. $\underline{024}$
LEVI, M. D. et al. Influence of ionic size on the mechanism of electrochemical doping of polypyrrole films studied by cyclic voltammetry. Electrochimica Acta, v. 42, n. 5, p. 757-769, 1997.

https://doi.org/10.1016/S00134686(96)00340-4

LI, X. G. et al. Novel multifunctional polymers from aromatic diamines by oxidative polymerizations. Chemical Reviews, v. 102, n. 9, p. 2925-3030, 2002. https://doi.org/10.1021/cr010423z

MERINO, E.; RIBAGORDA, M. Control over molecular motion using the cis - trans photoisomerization of the azo group. Beilstein J Org Chem., v. 8, p. 1071-90, 2012. https://doi.org/10.3762/bjoc.8.119

MOTIA, S. et al. Development of a novel electrochemical sensor based on electropolymerized molecularly imprinted polymer for selective detection of sodium lauryl sulfate in environmental waters and cosmetic products. Journal of Electroanalytical Chemistry, v. 823, n. March, p. 553-562, 2018. https://doi.org/10.1016/i.jelechem.2018.03. $\underline{069}$

MUCIO, M. et al. Sensors and Actuators B : Chemical Electrochemical sensor for dodecyl gallate determination based on electropolymerized molecularly imprinted polymer. Sensors \& Actuators: B. Chemical, v. 253, p. 180-186, 2017. https://doi.org/10.1016/j.snb.2017.06.127

OLEAN-OLIVEIRA, A.; TEIXEIRA, M. F. S. Sensors and Actuators $B$ : Chemical Development of a nanocomposite chemiresistor sensor based on $\pi$ - conjugated azo polymer and graphene blend for detection of dissolved oxygen. Sensors \& Actuators: B. Chemical, v. 271, , p. 353-357, 2018.

https://doi.org/10.1016/i.snb.2018.05.128 
PAULING, B. Y. L. A Theory of the Structure and Process of Formation of Antibodies $J$. Am. Chem. Soc. . v. 62, n. 10, p. 2643-2657, 1940. https://doi.org/10.1021/ja01867a018 PEREZ-RUIZ, F.; DALBETH, N.; BARDIN, T. A Review of Uric Acid, Crystal Deposition Disease, and GoutAdvances in Therapy, 2014. Adv Ther., v. 32, n. 1, p. 31-41. 2015 https://doi.org/10.1007/s12325-014-0175-z

REHAN, H. H. Electrosynthesis of conducting polymer films from the azo dye methoxy red. Journal of Applied Electrochemistry, v. 30, n. 8 p. $\quad 945-951, \quad 2000$. https://doi.org/10.1023/A:1004089413266

SEQUOIA, E.; GENIES, E. M.; TSINTAVIS, C. Redox mechanism and electrochemical polyaniline deposits behayiour of. Polymer, v. 195, p. 109-128, 1985. https://doi.org/10.1016/0022-

0728(85)80009-7

ALVES, L. T. O. et al. Síntese e caracterização de MIP com fenilalanina visando sua aplicação na técnica de SPE Synthesis and characterization of MIP with Phenylalanine for their application in SPE. v. 25, n. 6, p. 596-605, 2015. https://doi.org/10.1590/0104-1428.2116

TARLEY, C. R. T.; SOTOMAYOR, M. D. P. T.; KUBOTA, L. T. Polímeros biomiméticos em química analítica. Parte 1: preparo e aplicações de MIP (\&quot;Molecularly Imprinted Polymers\&quot;) em técnicas de extração e separação. Química Nova, v. 28, n. $6, \quad$ p. 1076-1086, 2005. https://doi.org/10.1590/S010040422005000600024

TEIXEIRA, M. F. S.; BARSAN, M. M.; BRETT, C. M. A. Molecular engineering of a $\pi-$ conjugated polymer film of the azo dye Bismarck Brown Y. RSC Adv., v. 6, n. 103, p. 101318-101322, 2016a. https://doi.org/10.1039/C6RA20335C
TEIXEIRA, M. F. S.; BARSAN, M. M.; BRETT, C. M. A. Molecular engineering of a piconjugated polymer film of the azo dye Bismarck Brown Y. RSC Adv., v. 6, n. 103, p. 101318, 2016b.

https://doi.org/10.1039/C6RA20335C

TORKASHVAND, M.; GHOLIVAND, M. B.; TAHERKHANI, $F$. Fabrication of an electrochemical sensor based on computationally designed molecularly imprinted polymer for the determination of mesalamine in real samples. Materials Science and Engineering C, v. 55, p. 209-217, 2015.

https://doi.org/10.1016/i.msec.2015.05.031

VOLKOV, A. et al. Electrochemical polymerization of aromatic amines. IR, XPS and PMT study of thin film formation on a Pt electrode. Journal of Electroanalytical Chemistry, v. 115, n. 2, p. 279-291, 1980. https://doi.org/10.1016/0368-

1874(80)80414-X

YANG, Y. et al. Materials Science \& Engineering C A photoresponsive surface molecularly imprinted polymer shell for determination of trace griseofulvin in milk. Materials Science \& Engineering C, v. 92, n. July, p. 365-373, 2018. https://doi.org/10.1016/i.msec.2018.06.069

YUAN, H. et al. Serum uric acid levels and risk of metabolic syndrome: A dose-response meta-analysis of prospective studies. Journal of Clinical Endocrinology and Metabolism, v. 100, n. 11, p. 4198-4207, 2015. https://doi.org/10.1210/ic.2015-2527

ZHENG, W. et al. Electrochemical sensor based on molecularly imprinted polymer / reduced graphene oxide composite for simultaneous determination of uric acid and tyrosine. Journal of Electroanalytical Chemistry, v. 813, p. 75-82, 2018. https://doi.org/10.1016/i.jelechem.2018.02. $\underline{022}$ 